\title{
Embedded Device Forensics and Security
}

\author{
KIM-KWANG RAYMOND CHOO, The University of Texas at San Antonio \\ YUNSI FEI, Northeastern University \\ YANG XIANG, Deakin University \\ YU YU, Shanghai Jiao Tong University
}

\begin{abstract}
While the increasing digitalization of our society and amalgamation of embedded devices into the everincreasing facets of our daily life (e.g., in smart and intelligent vehicles, smart cities and smart nations, and critical infrastructure sectors) have resulted in improved productivity and quality of life, the trend has also resulted in a trend of increasing frequency and sophistication of cyber exploitation and cyber threats. Hence, there is a need for coordinated efforts from the research community to address resulting concerns using both cryptographic and non-cryptographic solutions, such as those presented in this special section.
\end{abstract}

Categories and Subject Descriptors: C.2.2 [Security and Privacy]: Systems Security

General Terms: Applied Cryptography, Network Security

Additional Key Words and Phrases: Embedded device security, embedded device forensics, and digital forensics

\section{ACM Reference Format:}

Kim-Kwang Raymond Choo, Yunsi Fei, Yang Xiang, and Yu Yu. 2016. Embedded device forensics and security. ACM Trans. Embed. Comput. Syst. 16, 2, Article 50 (November 2016), 5 pages.

DOI: http://dx.doi.org/10.1145/3015662

\section{BACKGROUND}

Embedded devices play an important role in our increasingly interconnected and datacentric society, particularly in critical infrastructure sectors (e.g., smart grids), smart cities, and Internet-of-Things infrastructure [Bertino et al. 2016; Perera et al. 2015; Song et al. 2016]. However, these devices generally operate with limited power energy budget and work in a mobile (and potentially hostile) environment with limited connectivity. Thus, testing and debugging of such devices, such as those used in industrial software systems and cyber-physical systems, can be challenging in practice. For example, reproducing observed bugs can be challenging in environments where bug reports are often incomplete [Hu et al. 2016], systems are tightly coupled with their operating environment or are poorly observable, and embedded devices are resource constrained and offer limited support for advanced debugging mechanisms. Addressing such security challenges would require substantially extending current security solutions or rethinking the current security paradigms.

This special section is dedicated to reporting cutting-edge research on the topic of embedded device security and forensics, with a particular emphasis on novel techniques to secure embedded devices (including those found in cyber-physical systems) as well

Authors' addresses: K.-K. R. Choo, Department of Information Systems and Cyber Security, The University of Texas at San Antonio, San Antonio, TX 78249-0631, USA; email: raymond.choo@fulbrightmail.org; Y. Fei, Department of Electrical and Computer Engineering, Northeastern University, 360 Huntington Avenue, Boston, MA 02115, USA; email: yfei@ece.neu.edu; Y. Xiang, Deakin University, Centre for Cyber Security Research, 221 Burwood Highway, Burwood, VIC 3125, Australia; email: yang@deakin.edu.au; Y. Yu, Shanghai Jiao Tong University, Department of Computer Science and Engineering, Shanghai, China; email: yuyu@yuyu.hk.

Permission to make digital or hard copies of part or all of this work for personal or classroom use is granted without fee provided that copies are not made or distributed for profit or commercial advantage and that copies bear this notice and the full citation on the first page. Copyrights for third-party components of this work must be honored. For all other uses, contact the Owner/Author.

2016 Copyright is held by the owner/author(s).

1539-9087/2016/11-ART50

DOI: http://dx.doi.org/10.1145/3015662 
as obtaining evidential data from embedded devices in crimes that make use of sophisticated and secure technologies (e.g., the use of strong encryption to secure both data-at-rest and data-in-transit).

In this special section, a total of 12 submissions were accepted for publication. All submissions went through a rigorous peer-review process, which includes multiple follow-up rounds with the authors. We will now summarize these 12 accepted submissions in Sections 2 and 3, prior to discussing future research directions in Section 4.

\section{CRYPTOGRAPHIC SOLUTIONS AND ATTACKS}

Cryptographic solutions generally provide the capability to securely authenticate users and devices, for example, using access control and authenticated key exchange schemes [Boyd 2003; Choo 2009; Yang et al. 2015]. Thus, it is not surprising that in this special section, a number of the solutions proposed are cryptographic based, such as the bio-cryptographic fingerprint verification scheme presented by $\mathrm{Xi}$ et al. [2016]. ${ }^{1}$ This scheme binds cryptographic keys with fingerprint features. The authors then demonstrate that their scheme outperforms several existing bio-cryptographic techniques in terms of verification accuracy without sacrificing security and computational efficiency.

As noted in a survey of literature over a span of 15 years, Quick and Choo [2014] observe that data security, processing, and forensic technologies have not kept pace with data storage technologies. This is an observation shared by Puthal et al. [2016] in this special section, who then propose a dynamic key-length-based secure framework (DLSeF). The latter is designed to provide end-to-end security for big data stream processing. Using simulations, the authors demonstrate that DLSeF enjoys improved security processing time.

As previously noted, due to the resource constrained nature of embedded devices, there may be a need for a paradigm shift in designing embedded device security solutions, an observation shared by Yang et al. [2015], who suggested that "rather than focusing on designing lightweight and individual cryptographic primitives, security solution designers should consider taking a whole-of-system approach to achieve system/collective lightweightness, outsource expensive computations from resourceconstrained field devices to neighboring devices and equipment with more computational capacity, and selectively protect critical data (partial/selective protection of data of interest)." Similarly, in this special section, Song et al. [2016] presented a payment installment framework for drive-thru Internet in a vehicular ad hoc network (VANET) setting. The authors demonstrated that even in an environment where Internet connectivity is not stable, the proposed framework allows users to conduct secure transactions.

Also, in this special issue, Liu et al. [2016] examine the applicability of Optimal Prime Fields (OPFs), a special class of finite fields, in designing lightweight security solutions based on elliptic curve cryptography. The authors introduce an optimized arithmetic library for OPFs, which is resistant against Simple Power Analysis (SPA) attacks.

In addition to SPA attacks, differential fault analysis (DFA) and differential power analysis have also been shown to be effective in recovering cryptographic keys from embedded devices, including 3G/4G USIM Cards, as demonstrated by Liu et al. [2015]. In this special section, Fu et al. [2016] demonstrate how their DFA technique can be used to target ITUbee, a software-oriented block cipher for resource-constrained devices. Li et al. [2016], in this special issue, study blind fault attacks (BFA), which is another class of attacks that can be used to target embedded devices. Specifically,

\footnotetext{
${ }^{1}$ This article was unintentionally published as a regular article in TECS 15:4. http://dx.doi.org/10.1145/
} 2930669. 
they demonstrate the possibility of reducing the number of fault injections required in a BFA required to recover a cryptographic key.

Castiglione et al. [2016], in this special section, present a fragile reversible watermarking scheme designed to ensure the authenticity and integrity of functional magnetic resonance imaging (fMRI) images. The authors also explain how their proposed scheme can be integrated within fMRI systems equipment, via a prototype implementation on acredit card-sized single-board computer. Also, in a healthcare setting, Liu et al. [2016] present an approach that converts a secure attribute-based signature scheme into an efficient and secure online/offline attribute-based signature scheme that can be used to ensure the integrity of electronic health records and the anonymity of the data owner. In a different setting, Wang et al. [2016] present a secure synchronized reading system that is designed to hold multiple contests simultaneously in a cloud computing environment for electronic commerce purposes. In this system, the authors construct a randomness-reused identity based key encapsulation mechanism in order to support separable decapsulation. This allows the system to reduce both decryption delay and transmission delay.

Although quantum cryptography is not widely found and deployed in real-world applications (at the time of writing), the research community has already started studying post-quantum cryptography [Broadbent and Schaffner 2016; Chen et al. 2016]. In this special section, for example, Mozaffari-Kermani et al. [2016] noted that hash-, code-, and lattice-based solutions, as well as multivariate-quadratic-equations could potentially resist cryptanalysis efforts from pre-quantum and quantum computing devices. The authors then propose and evaluate fault diagnosis approaches for hash-based post-quantum signatures.

\section{NON-CRYPTOGRAPHIC APPROACHES AND FORENSICS}

Smart vehicles (e.g., Tesla smart cars) are becoming increasingly popular. These vehicles are equipped with various on-board and embedded devices, such as on-board computers, radio transceivers, sensors, and storage equipment. Given the nature of these vehicles and the real-world implications of a successful compromise of the system (e.g., real-world fatality due to an accident), it is important to ensure the security of data communications between the vehicle and the underlying infrastructure such as the city's intelligent transportation systems. In this special section, Gai et al. [2016] present a cloud-based model to provide secure real-time multimedia data sharing and transferring.

Mobile devices are another common consumer-oriented embedded device, and, given the (significant) amount of information that can be accessed and stored on such devices, it is important to understand the effectiveness of remote wiping and secure deletion apps and services on these devices. For example, Leom et al. [2016] recently reviewed both published literature and patents on existing approaches to secure flash storage deletion, as well as provided prototype experimental results for three Android devices. The findings from their review identified the lack of technical articles evaluating the implementation of remote wiping and secure deletion approaches or techniques on a wide range of popular mobile devices. This is the gap that Shu et al. [2016] seek to contribute to in this special section. Specifically, the authors reveal data erasure flaws in three typical scenarios on a selection of Android devices, and present an approach to forensically recover private data from the raw disk image without any file system information.

\section{CONCLUDING REMARKS}

Similarly to any consumer-oriented technologies, embedded devices will evolve and so will cyber exploitation of these devices in terms of attack vectors (e.g., vulnerabilities) 
and sophistication. As posited by Choo [2011, 2014] and Smith et al. [2004], cyber space cannot be simply shut down to address the threats due to malicious cyber activities. In order to mitigate emerging and evolving cyber security threats and make informed decisions about cyber and national security, it is important for us to look ahead and understand how embedded devices and other contemporary technologies can be exploited. While the articles in this special section have made significant contributions in embedded device security and forensics, much more work needs to be done along several directions, including the following:

- advanced security features for embedded devices and cyber-physical systems (e.g., computationally efficient anonymity and authentication schemes such as cancellable biometrics);

- cryptanalysis, side channel attacks, fault injection attacks, memory-based attacks, and other attacks targeting embedded devices and cyber-physical systems;

- forensic and anti-forensic techniques for embedded devices and cyber-physical systems;

- network traffic analysis and classification for traffic collected from embedded systems and cyber-physical systems, in an autonomous fashion;

- ontology of bug reproduction productivity tools for embedded devices and cyberphysical systems; and

- vulnerability, malware, and bug detection and mitigation techniques for embedded devices and cyber-physical systems.

\section{ACKNOWLEDGMENTS}

The authors thank the authors for their submissions, the reviewers for the invaluable and timely reviews, and the ACM Transactions on Embedded Compting Systems editorial board and administration, more specifically the Editor-in-Chief, Professor Sandeep K. Shukla, and Laura Lander and Jayel Tecson, from editorial support services, for their support. Without the support of the authors, reviewers, and the ACM TECS editorial board and administration, this special section would not have been possible.

\section{REFERENCES}

Elisa Bertino, Kim-Kwang Raymond Choo. 2016. Dimitrios georgakopolous, and surya nepal.internet of things (IoT): Smart and secure service delivery. ACM Trans. Internet Technol. 2016.

Colin Boyd. 2003. Protocols for authentication and key establishment. Information Security and Cryptography. Springer.

Anne Broadbent and Christian Schaffner. 2016. Quantum cryptography beyond quantum key distribution. Designs Codes Cryptogr. 78, 1 (2016), 351-382.

Arcangelo Castiglione, Raffaele Pizzolante, Francesco Palmieri, Barbara Masucci, Bruno Carpentieri, Alfredo De Santis, and Aniello Castiglione. 2016. On-board format-independent security of functional magnetic resonance images. ACM Trans. Embed. Comput. Syst. 2016.

Kim-Kwang Raymond Choo. 2009. Secure key establishment. Adv. Inform. Secur. 41, (2009).

Kim-Kwang Raymond Choo. 2011. The cyber threat landscape: Challenges and future research directions. Comput. Secur. 30, 8 (2011), 719-731, 2011.

Kim-Kwang Raymond Choo. 2014. A conceptual interdisciplinary plug-and-play cyber security framework. In ICTs and the Millennium Development Goals-A United Nations Perspective, H. Kaur and X. Tao, (Eds.). Springer, New York, 81-99.

Lily Chen, Stephen Jordan, Yi-Kai Liu, Dustin Moody, Rene Peralta, Ray Perlner, and Daniel Smith-Tone. 2016. Report on post-quantum cryptography. National Institute of Standards and Technology (NISTIR 8105).

Shan Fu, Guoai Xu, Juan Pan, Zongyue Wang, and An Wang. 2016. Differential fault attack on ITUbee block cipher. ACM Trans. Embed. Comput. Syst. 2016.

Keke Gai, Longfei Qiu, Min Chen, Hui Zhao, and Meikang Qiu. 2016. SA-EAST: Security-aware efficient data transmission for ITS in mobile heterogeneous cloud computing. ACM Trans. Embed. Comput. Syst. 2016. 
Yan Hu, Jun Yan, and Kim-Kwang Raymond Choo. 2016. PEDAL: A dynamic analysis tool for efficient concurrency bug reproduction in big data environment. Cluster Comput. 19, 1, 153-166, 2016.

Ming Di Leom, Kim-Kwang Raymond Choo, and Ray Hunt. 2016. Remote wiping and secure deletion on mobile devices: A review. J. Forens. Sci, 2016. DOI : 10.1111/1556-4029.13203

Beibei Li, Rongxing Lu, Wei Wang, and Kim-Kwang Raymond Choo. 2016. DDOA: A dirichlet-based detection scheme for opportunistic attacks in smart grid cyber-physical system. IEEE Trans. Inform. Forens. Secur. 11, 11, 2415-2425, 2016.

Yang Li, Zhe Liu, and Jian Wang. 2016. Reduction in number of fault injections for blind fault attack on SPN block ciphers. ACM Trans. Embed. Comput. Syst. 2016.

Jianghua Liu, Jinhua Ma, Wei Wu, Xiaofeng Chen, Xinyi Huang, and Li Xu. 2016. Protecting mobile health records in cloud computing: A secure, efficient, and anonymous design. ACM Trans. Embed. Comput. Syst. 2016.

Junrong Liu, Yu Yu, François-Xavier Standaert, Zheng Guo, Dawu Gu, Wei Sun, Yijie Ge, and Xinjun Xie. 2015. Small tweaks do not help: differential power analysis of MILENAGE implementations in 3G/4G USIM cards. In Proceedings of 20th European Symposium on Research in Computer Security (ESORICS 2015), Volume 9326/2015 of Lecture Notes in Computer Science. Springer-Verlag, 468-480.

Zhe Liu, Jian Weng, Zhi Hu, and Hwajeong Seo. 2016. Efficient elliptic curve cryptography for embedded devices. ACM Trans. Embed. Comput. Syst. 2016.

Mehran Mozaffari-Kermani, Reza Azarderakhsh, and Anita Aghaie. 2016. Fault detection architectures for post-quantum cryptographic stateless hash-based secure signatures benchmarked on ASIC. ACM Trans. Embed. Comput. Syst. 2016.

Charith Perera, Rajiv Ranjan, Lizhe Wang, Samee Ullah Khan, and Albert Y. Zomaya. 2015. Big data privacy in the internet of things era, IT Profess. 17, 3, 32-39, 2015.

Victor Prokhorenko, Kim-Kwang Raymond Choo, and Helen Ashman. 2016. Intent-based extensible realtime PHP supervision framework. IEEE Trans. Inform. Forens. Secur. 11, 10 (2016), 2215-2226.

Deepak Puthal, Surya Nepal, Rajiv Ranjan, and Jinjun Chen. 2016. DLSeF: A dynamic key length based efficient real-time security verification model for big data stream. ACM Trans. Embed. Comput. Systems, 2016.

Darren Quick and Kim-Kwang Raymond Choo. 2014. Impacts of increasing volume of digital forensic data: A survey and future research challenges. Dig. Invest. 11, 4 (2014), 273-294.

Junliang Shu, Yuanyuan Zhang, Juanru Li, Bodong Li, and Dawu Gu. 2016. Why data deletion fails? A study on deletion flaws and data remanence in android systems. ACM Trans. Embed. Comput. Syst. 2016.

Russell G. Smith, Peter Grabosky, and Gregor Urbas. 2004. Cyber Criminals On Trial. Cambridge University Press, Cambridge.

Jun Song, Chunling Han, Kaixin Wang, Jian Zhao, Rajiv Ranjan, and Lizhe Wang. 2016. An Integrated Static Detection and Analysis Framework for Android, Pervasive and Mobile Computing, 2016.

Jun Song, Fan Yang, Kim-Kwang Raymond Choo, Zhijian Zhuang, and Lizhe Wang. 2016. SIPF: A secure installment payment framework for drive-thru internet. ACM Trans. Embed. Comput. Syst. 2016.

Kai Xi, Jiankun Hu, and B. V. K. Vijaya Kumar. 2016. FE-SViT: A SViT based fuzzy extractor framework. ACM Trans. Embed. Comput. Syst. 15, 4 (2016), Article 78.

Wei Wang, Peng Xu, Laurence Tianruo Yang, Willy Susilo, and Jinjun Chen. 2016. Securely reinforcing synchronization for embedded online contests. ACM Trans. Embed. Comput. Syst. 2016.

Yanjiang Yang, Jiqiang Lu, Kim-Kwang Raymond Choo, and Joseph K. Liu. 2015. On lightweight security enforcement in cyber-physical systems. In Proceedings of 4th International Workshop on Lightweight Cryptography for Security and Privacy (LightSec'15), Volume 9542/2015 of Lecture Notes in Computer Science. Springer-Verlag, 97-112.

Yanjiang Yang, Joseph K. Liu, Kaitai Liang, Kim-Kwang Raymond Choo, and Jianying Zhou. 2015. Extended proxy-assisted approach: achieving revocable fine-grained cloud data encryption. In Proceedings of 20th European Symposium on Research in Computer Security (ESORICS'15), Volume 9327/2015 of Lecture Notes in Computer Science. Springer, Verlag, 146-166.

Received October 2016; revised November 2016; accepted November 2016 